\title{
Utility of electroencephalogram in altered states of consciousness in intensive care unit patients
}

\author{
F. N. Kapadia, S. Vadi, U. Shukla, R. Gursahani
}

\begin{abstract}
Background: EEG is an investigative tool for assessing cerebral activity. Although certain EEG patterns may have a specific diagnostic or prognostic inference, they may not be precise for any sole etiology in majority of cases and may need clinical correlation. Objective: Aim of this study was to assess the severity and prognosis of cerebral dysfunction in patients admitted to Intensive Care Unit (ICU) and to evaluate the incidence of non-convulsive status epilepticus (NCSE). Design: A prospective study, wherein we analyzed EEG characteristics in a series of 70 patients. Setting: A tertiary care hospital in Mumbai, India. Patients: EEG characteristics of 70 patients admitted in ICU over a period of 9 months were comprehensively analyzed. These patients were clinically examined and a questionnaire was completed without knowledge of the EEG findings. EEGs were requested for by neurologist or intensivist and our inclusion criteria were (i) patients with altered sensorium of varying etiology, (ii) unconscious patients at risk for non-convulsive status epilepticus (those with a history of epilepsy), and (iii) unconscious patients with involuntary jerky eye movements. Results: Of the various clinical presentations on ICU admission, there were 20 patients with seizures, 15 with metabolic disorders, 13 with infective causes, 9 with hypoxia, 9 with cerebro-vascular accident on presentation, 1 patient with alcohol/drug overdose, 2 with intra-cerebral space occupying lesion and 1 with ambiguous etiology on admission (there being an overlap among the presentation). Mean duration from presentation to performing EEG was 13 hours. 64 (91.42\%) patients had abnormal EEGs. 32(50\%) patients had EEG slowing and 4(6.25\%) patient had electro cerebral inactivity. Eleven (21.87\%) patients had epileptiform activity on the EEG of which seven did not have overt seizures (NCSE). Follow-up EEGs of these patients showed resolution of the epileptiform activity. Conclusions: EEG is useful in patients admitted to ICU in diagnosing NCSE and various other conditions. Emergent EEG study in obtunded patients provides valuable diagnostic and prognostic information.
\end{abstract}

Key Words: EEG, altered levels of consciousness, Non-convulsive status

\section{Introduction}

EEG monitoring in ICU is done to assure cerebral wellbeing, as an accomplice to neurological examination. EEG confirms not only that the seizures are occurring but also the nature and the frequency of these episodes. This study is also helpful in the interictal period, during

\section{From:}

P. D. Hinduja National Hospital and Medical Research Center, Mumbai, India Correspondence:

Vadi S,

P. D. Hinduja National Hospital and Medical Research Center, Mahim, Mumbai 400016, India. E-mail: sonalivadi@hotmail.com which certain electrical cerebral abnormalities suggest epilepsy.

Status epilepticus could either present as convulsive, partial status or as a non-convulsive status. Non-convulsive status has no overt tonic-clonic activity and presents as an altered level of consciousness, sometimes coma. Clinically, the patient in NCSE may have abnormal ocular movements. ${ }^{[1]}$ NCSE can occur with several illnesses. These include hypoxic brain damage, metabolic abnormalities or following a convulsive epi-

Free full text available from www.ijccm.org 
sode. ${ }^{[2]}$ Patients who are known epileptics are also at a risk for NCSE. ${ }^{[3]}$ The usual EEG characteristics of nonconvulsive status epilepticus (NCSE) are either focal rhythmic or semi-rhythmic slow or sharp waves superimposed on a slow background.

NCSE also is associated with a high morbidity and mortality and hence the diagnosis has to be made urgently keeping a high index of suspicion. ${ }^{[4,5]}$ We conducted this study to analyze the utility of EEG in ICU patients and to evaluate the incidence of non-convulsive status.

\section{Materials and Methods}

This was a prospective study conducted in the ICU of a tertiary care center in Mumbai over a period of 9 months (May 2003 to Jan 2004). Patients were clinically evaluated in detail (that included duration of symptoms, presence of overt seizures, Glasgow Coma Scale, whether the patient was on anti-epileptic drugs and any abnormal laboratory values) and a questionnaire was completed by the medical residents without knowledge of the EEG findings. EEGs were requested for by either intensivist or the neurologist. Inclusion criteria adopted for this study were patients with altered sensorium of varying etiology, unconscious patients at risk for NCSE (i.e. those with a history of epilepsy) and unconscious patients with subtle tonic-clonic/jerky eye movements.

\section{Results}

There were a total of 70 patients. Various etiologies for admission to the ICU with altered sensorium are shown in Figure 1. The mean age was 50.2 years (range 10-73 years). Male to female ratio was 1:1.1. Mean duration from presentation to performing an EEG was 13 hours. In all 14 (20\%) patients died. Of these were 5/9
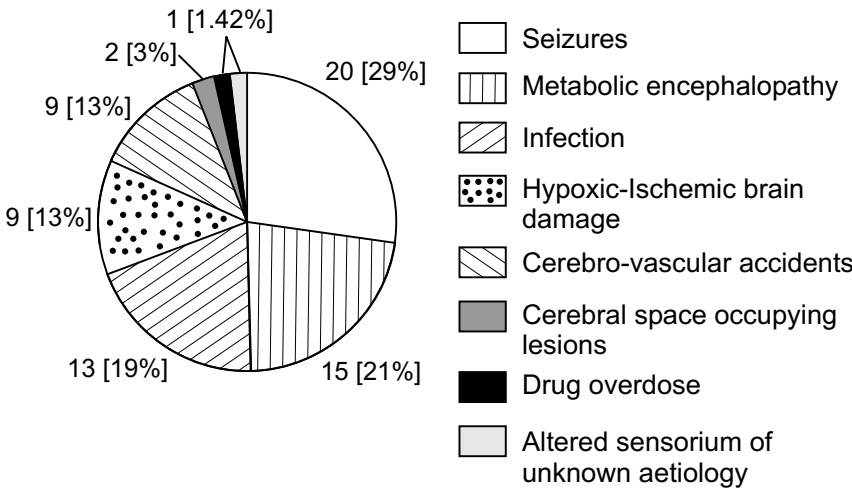

Figure 1: Etiology of altered sensorium of the patients admitted in the ICU
(7.14\%, P: 0.004) patients with hypoxia, 4/15 (5.71\%, $P: 0.21)$ patients with metabolic cause on presentation, $2 / 13(2.85 \%, P: 0.57)$ had an infective etiology, $2 / 20$ $(2.85 \%, P: 0.22)$ patients had overt seizures and $1 / 9$ $(1.42 \%, P: 0.77)$ patient with cerebro-vascular accident on presentation.

Table 1 shows the EEG patterns observed [Figure 2 and 3]. Patients with epileptiform activity on EEG ( $n=11)$ included 4 with overt seizures and the remaining 7 patients were in non-convulsive status [Figure 4]. Further analyzing these patients with NCSE, mean age of these patients was 53 years (range 25-66 years). Male to female ratio was 1:1.3. Four of the patients had infective etiology on presentation to the ICU, and one each was of hypoxic brain damage following head injury, metabolic encephalopathy and cerebral space occupying lesion. Two patients had a Glasgow coma scale (GCS) of $<8,4$ patients had a GCS of 9-12 and 1 patient had a GCS of 13-15. None of these patients had jerky eye movements.

Two patients (28.57\%) with NCSE expired during their ICU stay.

When investigating the other EEG abnormalities, fo-

\begin{tabular}{lc}
\hline Table 1: EEG patterns on presentation to ICU \\
\hline EEG findings & No. of patients \\
Generalized slowing & 32 \\
Epileptiform activity & 11 \\
Normal & 6 \\
Low amplitude & 6 \\
Post-ictal slowing & 6 \\
Focal slowing & 5 \\
No detectable activity & 4 \\
\hline
\end{tabular}

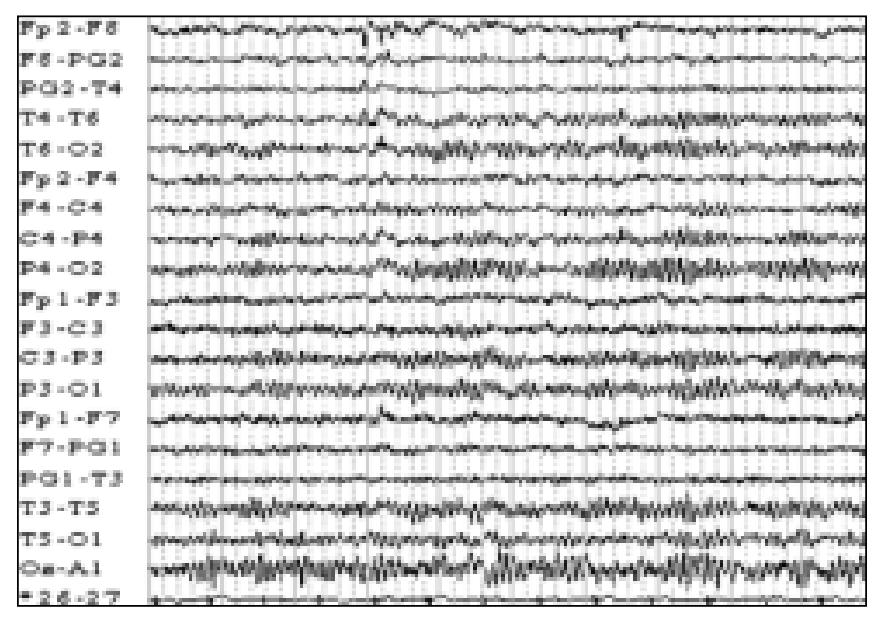

Figure 2: Fast Beta waves, Focal discharges and Alpha waves 


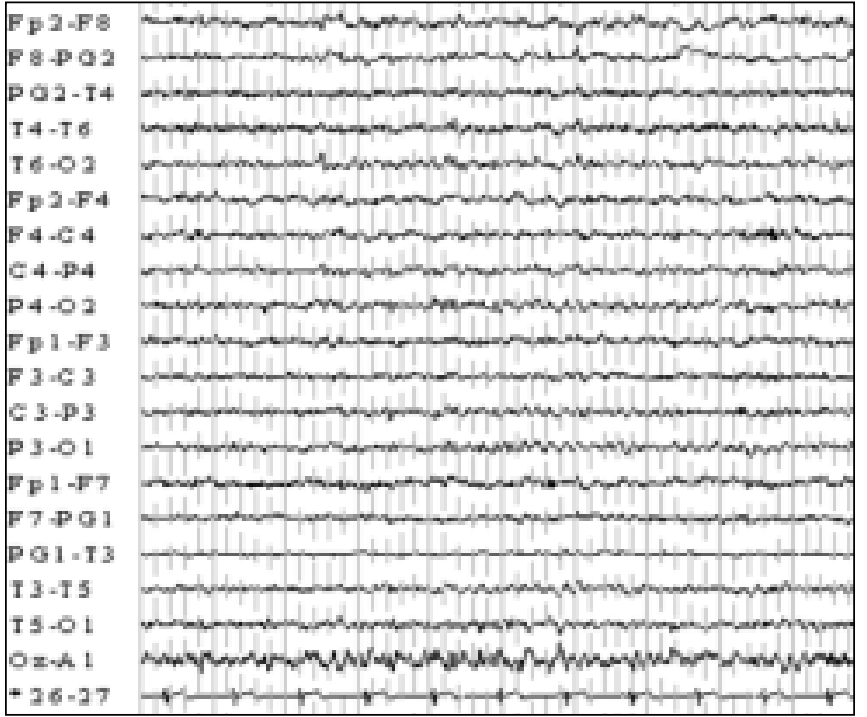

Figure 3: Delta waves:

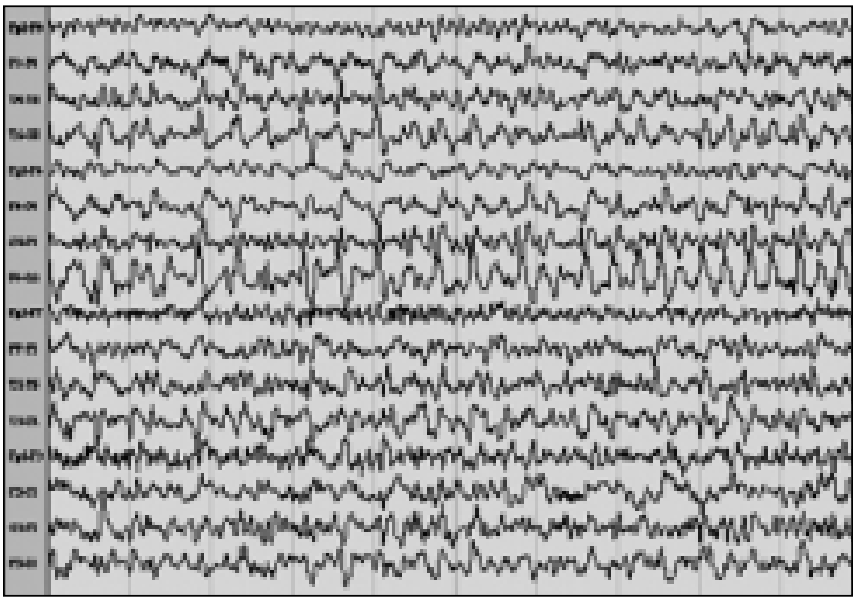

Figure 4: EEG recording of NCSE: Intermittent spike waves seen on the temporal and parieto-occipital regions

cal EEG slowing correlated with CT scan findings. EEGs with no detectable activity correlated clinically (i.e.) brain dead. $50 \%(n=3 / 6)$ patients with low amplitude EEG died. Thirty-two patients had generalized slowing on EEG of whom 2/14 (14.28\%) patients having reversible metabolic cause on admission to ICU died and $100 \%$ ( $n=0$ ne) patient with irreversible metabolic cause (hypoxicischemic brain damage) on presentation died.

\section{Discussion}

NCSE is an under-recognized cause of coma. It is difficult to diagnose NCSE in obtunded patients as they also have underlying medical co-morbidities. They may have subtle sporadic movements or may have no overt convulsions at all. Again, patients in ICU are often sedated and paralyzed and it is difficult to detect clinically any seizure activity in them, EEG thus being helpful in identifying seizure activity and the effectiveness of treatment. Thus, EEG should be routinely performed for evaluating comatose patients even if no obvious seizure activity is found.

In this study, the incidence of NCSE was $10 \%$. Patients with EEG patterns showing low amplitude and those with electro cerebral inactivity had poor prognosis. A literature review revealed the incidence of NCSE to vary from $10.7-19 \%,{ }^{[6,7]}$ which is similar to our observation.

Highest mortality was found in patients with hypoxicbrain damage (generalized slowing on EEG) $10 \%$, followed by $33.33 \%$ in patients with post-ictal slowing, $28.57 \%$ mortality in patients with epileptiform activity without overt seizures (NCSE) and $14.28 \%$ mortality in patients with reversible metabolic cause (generalized slowing on EEG) on presentation.

\section{Conclusion}

Electro-clinical correlation allowed NCSE to be diagnosed and treated in seven (10\%) patients admitted to the ICU. In addition, EEG abnormalities could also be of value in determining the outcome of patients with treatment.

\section{References}

1. Husain AM, Horn GJ, Jacobson MP. Non-convulsive status epilepticus: Usefulness of clinical features in selecting patients for urgent EEG. J Neurol Neurosurg Psychiatry 2003;74:189-91.

2. Brenner RP. Is it status? Epilepsia 2002;43(Suppl 3):103-13.

3. Khan RB, Yerremsetty PK, Lindstrom D, McGill LJ. Emergency EEG and factors associated with nonconvulsive status epilepticus. J Natl Med Assoc 2001;93:359-62.

4. Kaplan PW. Assessing the outcomes in patients with nonconvulsive status epilepticus: Nonconvulsive status epilepticus is underdiagnosed, potentially overtreated, and confounded by comorbidity. J Clin Neurophysiol 1999;16:341-52.

5. Ruegg SJ, Dichter MA. Diagnosis and treatment of Non Convulsive Status Epilepticus in an ICU setting. Curr Treat Options Neurol 2003:5:93-110.

6. Varelas PN, Spanaki MV, Hacein-Bey L, Hether T, Terranova B. Emergent EEG: Indications and Diagnostic yield. Neurology 2003;61:702-4.

7. Claassen J, Mayer SA, Kowalski RG, Emerson RG, Hirsch LJ. Detection of electrographic seizures with continuous EEG monitoring in critically ill patients. Neurology 2004; 62:1743-8. 